\title{
nature
}

13 November 2003 Volume 426 Issue no 6963

\section{Towards a clean collider}

The high-energy physics community has grand plans to probe deeper into the structure of matter and space-time. The proposal for a multinational linear collider merits strong support.

A lthough the Large Hadron Collider (LHC) is still years away from completion at CERN in Geneva, the particle-physics community is already mobilizing to push through the funding for its next project - a US\$6-billion linear collider to study collisions between electrons and anti-electrons. The announcement by the US Department of Energy (DOE) on Monday of its funding priorities for scientific facilities over the next 20 years is grist to their mill: the proposed Linear Collider ranks 13th out of 28 projects, in a list headed by the fusion experiment ITER (see page 108). The Linear Collider is classed first among the 'mid-term' priorities - mid-term being an indication that construction of this facility is still some way off.

But plans are progressing. In Paris next week, a steering committee will meet to establish a panel of 'wise men' to oversee the choice of accelerator technology for what is usually known as the International Linear Collider (ILC). Throughout 2004, the panel will review four competing accelerator designs, all at varying stages of development in laboratories around the world. By the end of the year, they will make their choice, and an international design project will be founded.

The particle-physics community is well-practised at such largescale cooperation. CERN, established by nine European countries and now counting 20 European states as full members, celebrates its 50th birthday next year, marking a remarkable period of collaborative effort that has made possible research that no one country could itself afford to pursue. By financial necessity, the LHC project has expanded to include 'guest' states, including the United States, India and China. So the step to a formal international project is small, and the will is great. Speaking at a conference this summer, Peter Rosen of the DOE stressed that the ILC project must be an international effort "from inception". A web-based petition in support of the ILC has been signed by more than 1,800 particle physicists worldwide.

The standard model of particle physics has been explored and verified more stringently than perhaps any other model in science. Occasional hints of something unexplained, something beyond the standard model, have evaporated. But the standard model is not the whole story - there are still issues to be resolved, such as the existence of the Higgs boson. The search for this elusive particle has been promoted as a major justification for building the LHC and for a substantial upgrade of Fermilab's Tevatron accelerator.

\section{Another dimension}

What more can the ILC offer? The new machine, planned to stretch over 30 kilometres, will give physicists the chance to look beyond the discovery of the Higgs boson. This particle is likely to appear in the high-energy proton-proton collisions in the LHC. But that environment is 'messy': to pick out in fine detail the true nature of this particle (or particles, as it may be one of many), the precise, clean physics of the ILC's electron-anti-electron collisions will be necessary. The interplay between the LHC and the ILC is a central plank in the physics case. The ILC won't just be tidying up after the LHC, but, if built quickly enough, it will feed information back into refined analyses at the LHC.
There is likely to be more to investigate at the energy scales accessible to the ILC. For example, there may be another family of particles that mirrors the fundamental particles of the standard model. These are 'supersymmetric' particles, the lightest of which is a prime candidate for the dark matter in the Universe. As with the Higgs boson, the essential nature of this postulated particle should become clear at the ILC. There may also be extra dimensions of space, beyond the three of familiar experience: data from the ILC will tell us how many and how big they are.

The ILC will increase the energy of its collisions in stages. This makes sense from a design perspective, but will also bring a degree of flexibility to this project that has been impossible elsewhere. The ILC should be able to 'home in' on a particular collision energy, with the result, say, that copious amounts of Higgs bosons could be generated for study, or that a certain region of phase space in which the LHC has thrown up some anomaly can be investigated further. The machine could also be adapted at a later stage so that, instead of electrons and their anti-particles, the colliding particles are photons. Again this is another window on Higgs physics, and more besides.

\section{Strong record}

The United States will be expected to be a major player in the ILC project, and may even host the machine itself. Despite the DOE's endorsement - and putting the issue of visas for visiting scientists aside - the situation in the United States is still complicated by the spectre of the Superconducting Supercollider, a US\$3-billion enterprise whose demise in 1993 shook the confidence of US particle physicists. Over the summer, that confidence was undermined once more, as reports from Fermilab suggested that its Tevatron accelerator may not deliver what was promised.

Meanwhile, in Europe, German physicists are still reeling from the halting of their bid to host a linear collider, which was a victim of a poor economic climate in that country. And the LHC project at CERN has suffered its own well-publicized problems, which resulted in a full external review last year. But those lessons have been learned well: far from being off-putting, that experience bodes well for the future. This physics community has, overall, a strong record in the management and delivery of accelerator-based projects, and its level of organization should inspire confidence in its ability now to deliver the ILC. Nevertheless, CERN may well be eyeing the ILC with caution: the laboratory's LHC debts will not be paid off until 2010 , and its management will be wary of any project that might lure away additional funds .

Physicists in the United States must now ensure that the ILC keeps its place on that DOE plan — which comes with the caveat that it will be reassessed periodically and its priorities may change. Worldwide, physicists must convince their politicians that the grand scheme of the Large Hadron Collider, followed by an International Linear Collider, offers a vital and vibrant programme of fundamental physics. Perhaps the possibility of spin-off technology from the development of the linear collider will tip the balance. With the estimated \$6-billion cost of the ILC spread over several years and shared by many nations, this is a bargain too good to miss. 